\title{
Integration of Electric Vehicles in the Electric Power System
}

\author{
A conceptual framework for integrating electric vehicles into electric power systems \\ is given; impacts and benefits arising from their use are discussed. \\ By João A. Peças Lopes, Senior Member IEeE, Filipe Joel Soares, Student Member IEEe, And \\ Pedro M. Rocha Almeida, Student Member IEEE
}

\begin{abstract}
This paper presents a conceptual framework to successfully integrate electric vehicles into electric power systems. The proposed framework covers two different domains: the grid technical operation and the electricity markets environment. All the players involved in both these processes, as well as their activities, are described in detail. Additionally, several simulations are presented in order to illustrate the potential impacts/benefits arising from the electric vehicles grid integration under the referred framework, comprising steady-state and dynamic behavior analysis.
\end{abstract}

KEYWORDS | Aggregator; ancillary services; electric vehicle; energy storage; hierarchical control; microgrid; multimicrogrid; vehicle-to-grid

\section{NOMENCLATURE}

EV Electric Vehicle.

V2G Vehicle-to-Grid.

RES Renewable Energy Sources.

MV Medium Voltage.

DSO Distribution System Operator.

LV Low Voltage.

CAU Central Aggregation Unit.

Manuscript received October 30, 2009; revised July 14, 2010; accepted July 24, 2010. Date of publication October 4, 2010; date of current version December 17, 2010. This work was supported in part by Fundação para a Ciência e Tecnologia under Grants SFRH/BD/48491/2008 and SFRH/BD/47973/2008 and within the framework of the Project Green Island with the Reference MIT-PT/SES-GI/0008/2008, by Fundo de Apoio à Inovação (Ministério da Economia, da Inovação e do Desenvolvimento), within the framework of the Project REIVE-Redes Eléctricas Inteligentes com Veículos Eléctricos, and by the European Union within the framework of the European Project MERGE-Mobile Energy Resources in Grids of Electricity, Contract 241399 (7th Framework Programme).

The authors are with the Instituto de Engenharia de Sistemas e Computadores do Porto - INESC Porto, 4200-465 Porto, Portugal and also with the Faculdade de Engenharia da Universidade do Porto-FEUP, Porto, Portugal (e-mail: jpl@fe.up.pt; fsoares@inescporto.pt; pedro.almeida@fe.up.pt).

Digital Object Identifier: 10.1109/JPROC.2010.2066250

\author{
MGAU Microgrid Aggregation Unit. \\ HV High Voltage. \\ VC Vehicle Controller. \\ CVC Cluster of Vehicles Controller. \\ GENCO Generation Company. \\ TSO Transmission System Operator. \\ MG Microgrid. \\ MMG Multimicrogrid. \\ MGCC Microgrid Central Controller. \\ CAMC Central Autonomous Management Controller. \\ DMS Distribution Management System. \\ AGC Automatic Generation Control.
}

\section{INTRODUCTION}

The future solution for the fossil fuels scarcity, as well as to the environmental problems associated with their wide usage, will most likely involve an extensive use of EVs. Currently, there are three relevant types of electric vehicles prepared to be launched in the markets: fully EVs, fuel cell EVs, and hybrid EVs [1], [2]. Battery and fuel cell EVs are driven only by electric power while current available hybrid EVs have also an internal combustion engine. Since these vehicles will require the use of batteries with high energy storage capacity and with large electric load charging requirements, a large deployment of this concept will provoke considerable impacts in electric power system design and operation, but will also enable and benefit the usage of nonpollutant energy resources [3], [4]. In [5] and [6], it is shown how EVs will likely change the energy demand, while in [7], the economic impacts for the electrical utilities are presented. In [8], the challenges for the rollout of EVs are described, including battery technology and cost, infrastructure financing, regulatory requirements, electrical power sector interface, and consumer acceptance issues. Finally, in [9], besides the changes in 
demand patterns, grid technical impacts are also addressed, namely concerning substation transformer loading and influence of temperature in their expected life.

When parked and plugged into the electric grid, EVs will absorb energy and store it, being also able to deliver electricity back to the grid. The latter is the distinctive feature of the V2G concept, allowing the provision of several ancillary services like peak power and spinning reserves [1]. In order to be able to provide these services, each EV must have some extra equipment like an electronic interface for grid connection, to allow controlled electric energy exchanges, a meter device, and a bidirectional communication interface to communicate with an aggregator entity, which will be in charge of managing a high number of EVs, as is described in [10].

A large deployment of EVs will involve:

- evaluation of the impacts that battery charging may have in system operation;

- identification of adequate operational management and control strategies regarding batteries' charging periods;

- identification of the best strategies to be adopted in order to use preferentially RES to charge EVs;

- assessment of the EV potential to participate in the provision of power systems services, including reserves provision and power delivery, within a V2G concept.

In what concerns ancillary services provision, EVs may be capable of supplying extra power for peak load demand, performing load shifting at distribution level or providing either spinning reserves or regulation to the system, namely if islanded operation is envisaged for certain areas of the distribution grid.

To accurately evaluate the impact of this new load/ storage devices, several deployment scenarios need to be studied taking into account several variables like the type of vehicles (fleet or individual), the technology used (electric, hybrid, or fuel cell), the owners' behavior, the traffic patterns, the places where cars are parked and connected to the grid, the type of connection between the $\mathrm{EV}$ and the network, as well as the grid control architecture.

The replacement of conventional vehicles by EVs will also require specific local charging infrastructures. Several solutions may arise to fit different EV owners' needs, namely:

- charging stations dedicated to fleets of EVs;

- fast charging stations;

- battery swapping stations;

- domestic or public individual charging points for slower charging.

All cases need to be considered when addressing the problems that will result from this future shift in the mobility paradigm. However, in the work presented in this paper, only slow charging at home and in public charging points located in residential areas is considered.
It is expected that large-scale penetration of EVs will increase electricity consumption, during charging periods. Therefore, power flows, grid losses, and voltage profile patterns along the grid will change considerably. Additionally, EV capability to provide energy to the system will also impact the grid flows. The combination of all these effects might oblige to reinforce the grid at some locations. Nonetheless, depending on the EV charging strategy to be adopted, reinforcement postponements may be achieved.

It is also expected that the amount of intermittent RES that can be safely integrated into the electric power system may increase due to EV storage capacity [11]. Given the uncontrollability of these energy sources, since they only produce energy when the primary renewable resource is available, EV capability to store energy and inject it later into the system will avoid spillage of "clean" energy, resulting in the decreased usage of the conventional fossil fuel units and expensive generators during peak hours [12]. This will allow reducing pollutants' emissions [12] and energy generation costs, provoking also strong impacts in the electricity markets behavior [13].

Recent researches [11], [14]-[16] have been conducted in order to provide new mechanisms that make EV integration simpler and beneficial to EV owners, utilities and, in a more general approach, the environment.

This paper describes a conceptual framework capable of dealing, in the future, with the grid technical management and market operation, in order to handle EV charging in an effective manner. To prove the efficiency of the suggested grid control architecture and market framework, two case studies are presented, where comparisons on grid operation performance are made confronting advanced control EV charging strategies and uncontrolled charging approaches. The first case study addresses the impacts of EVs in an MV network, as well as the benefits for the DSO arising from the adoption of a smart charging approach. The second case study addresses the impacts of EVs in the dynamic behavior of a small LV grid and of a larger MV grid, both operated in islanded manner.

\section{GRID CONTROL ARCHITECTURE AND MARKET FRAMEWORK}

The technical management of an electric power system having a large-scale deployment of EVs will require, for their battery charging, a combination of:

- a centralized hierarchical management and control structure;

- a local control located at the EV grid interface.

In fact, the simple use of a smart EV grid interfacing device does not solve all the problems arising from EV integration in distribution networks. These interfaces can be rather effective when dealing with voltage drops originated by EV charging, by locally decreasing charging rates through a voltage droop control approach, but fail when addressing issues that require a higher control level, 
such as managing branches' congestion levels or enabling EVs to participate in the electricity markets. For these cases, coordinated control is required and so a hierarchical management and control structure responsible for the entire grid operation, including EV management, must be available. Therefore, the efficient operation of such a system depends on the combination of local and centralized control modes. The latter control approach relies on the creation of an adequate communications infrastructure capable of handling all the information that needs to be exchanged between the entities of the hierarchical control structure and the EV.

When operating the grid in normal conditions, EVs will be managed and controlled by a new entity-the aggregator-whose main functionality will be grouping EVs, according to their owners' willingness, to exploit business opportunities in the electricity markets.

However, due to high uncertainties related to when and where EV owners will charge their vehicles, the existence of a grid monitoring structure will be required, controlled by the DSO, with the capability of acting over EV charging in abnormal operating conditions, i.e., when the grid is being operated near its technical limits, or in emergency operating modes, e.g., islanded operation. In these situations, EVs might receive simultaneously two different set points: one from the aggregator and other from the monitoring and management structure headed by the
DSO. To avoid violation of grid operational restrictions, the DSO signals will override the aggregator ones.

The technical and market management proposed structures are described in Fig. 1. The column on the right-hand side describes the market operation, whereas in the left-hand side the technical operation framework is explained. This control and management structure is able to handle the previously exposed type of operating conditions.

\section{A. Normal System Operation}

As stated above, in order to manage a large amount of EVs parked in a large geographical area, where MV and LV grids exist, the existence of aggregators will be necessary that will serve as interface between EVs and electricity markets. These aggregators will have the capability of grouping EVs so that together they represent a load/storage device with the adequate size to participate in electricity markets, in a similar way as described in [10].

Given the complexity of the information that an aggregator needs to collect and process, a hierarchical management structure, independent from the DSO, is suggested in this paper (Fig. 1). Since each aggregator is responsible for a large geographical area, it will be composed by two different types of entities: the CAU and the MGAU. The CAU is an entity that takes care of a maximum of 20000 costumers that are fed by each HV/MV substation,

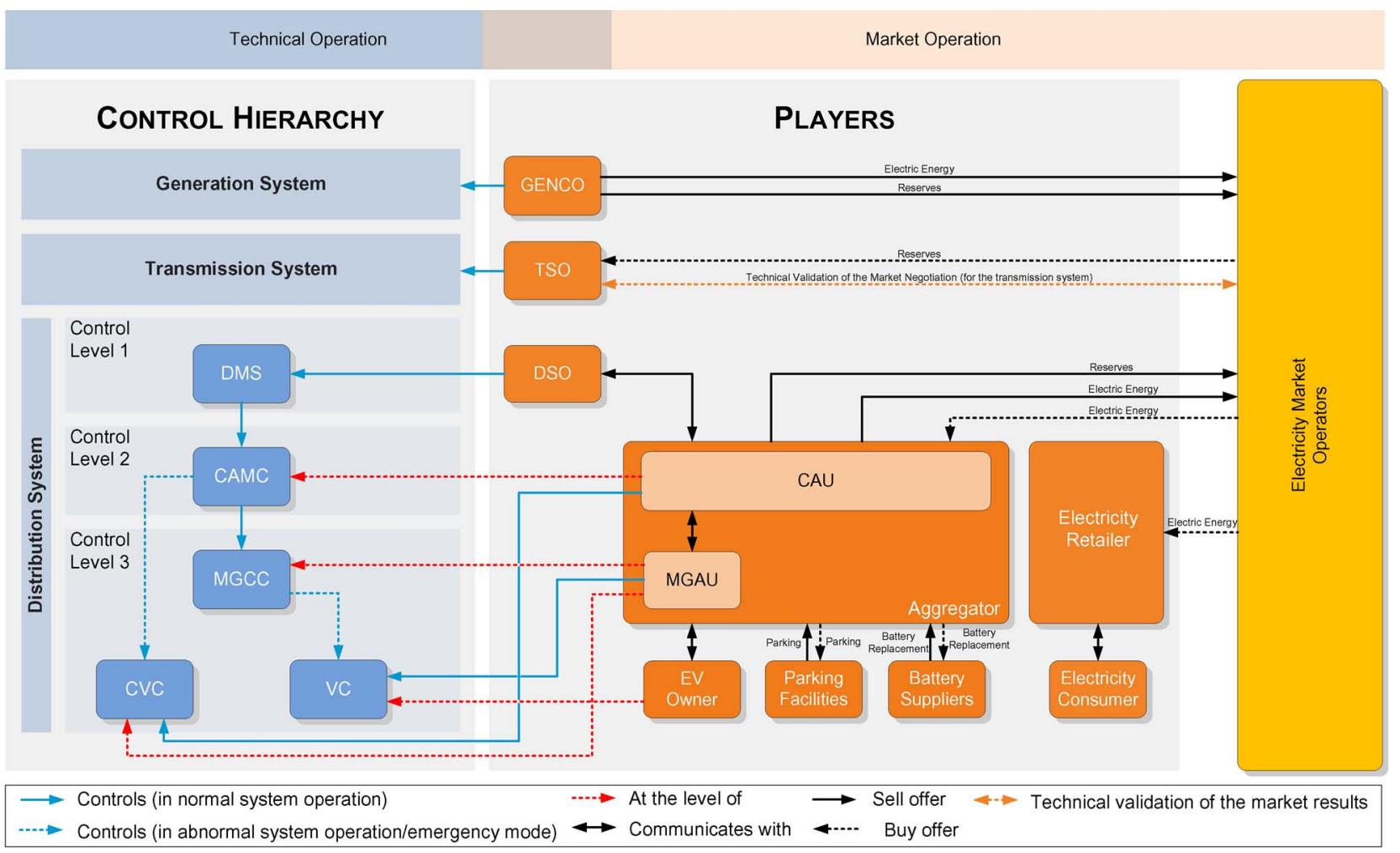

Fig. 1. Technical management and market operation framework for EV integration into electric power systems. 
communicating with several downstream MGAU which, by their turn, will be located at the MV/LV substation level, with an expected maximum of about 400 costumers each. The MGAU was created in order to decrease communications and computational burden that a real implementation of the concept would require, providing $\mathrm{CAU}$, the core unit of the aggregator, already preprocessed information regarding groups of EVs located in the LV grids. Each EV must have a specific interface unit-the $\mathrm{VC}$ - to enable bidirectional communication between the $\mathrm{VC}$ and the upstream charging management system. In addition to the $\mathrm{VC}$, there is a new type of element, the CVC, designed to control the charging of large parking lots (e.g., shopping centers), and fed directly from the MV network. Individual controllers of EVs under a CVC management do not have an active VC communicating with higher hierarchical controllers. For normal operation, the VC will interact with the MGAU and the CVC directly with the CAU.

Based on historical data, the aggregators will forecast the market behavior for the next day and will prepare their buy/sell bids. Having this defined, a prior negotiation with the DSO must exist to prevent the occurrence of severe congestion and voltage problems in the distribution networks. The aggregators will present their day-ahead proposal to the DSO, which will analyze it to evaluate its technical feasibility. If valid, the aggregator can proceed to the market negotiation. If not, the DSO will ask the aggregator to make the changes needed to guarantee a safe operation of the distribution grid on the next day. It is foreseeable that in this case the DSO will have to compensate the aggregator by this service. The DSO might even request the aggregator to change further its plans in order to decrease the energy losses in the distribution network.

If the market prices of electricity are cost reflective (i.e., include the cost of electricity generation, transmission, and distribution), a direct consequence of the hourly energy prices variation will be the flattening of the daily load diagram. As a response to the energy prices, aggregators will naturally perform load shifting in order to provide energy at a lower cost to their clients. They will buy electricity from the market mainly during the night, at lower prices, to charge their clients' EVs, and they may sell it during the day, at peak hours, taking advantage of their clients' EV storage capability. Aggregators will compete directly with electricity retailers for energy acquisition and with GENCO for selling energy.

Taking advantage of EV capability to provide reserves, EVs might offer also in the electricity markets this system's services to the TSO, competing once again with the GENCO.

After market closure, the TSO proceeds to the evaluation of the load/generation schedules and, if problems on the transmission system are foreseen, it requests modifications to these schedules until feasible operating conditions are attained.
Every day the aggregator will manage the EVs under its domain, according to what was previously defined in the market negotiations and validated by the TSO, by sending set points to $\mathrm{VC}$ or CVC related with rates of charge or requests for provision of ancillary services. To accomplish successfully such a complex task, it is required that every fixed period (likely to be defined around $15 \mathrm{~min}$ ), the state of charge of each EV battery is communicated to the aggregator, to assure that, at the end of the charging period, batteries will be charged according to EV owners' requests.

Parking and new battery supplying are also services that aggregators can negotiate in other markets, as mentioned in [10] and included in Fig. 1. Nonetheless, these parallel markets' negotiations will not be addressed in this paper.

Yet, other research paths have developed slightly different approaches to deal with EV integration, such that commercial and technical responsibilities are managed in an integrated way [16]. This requires concentrated knowledge on both state of charge at the connection moment and completion time for each EV as well as network technical and load profile data.

\section{B. Abnormal System Operation or Emergency Mode}

When grid normal technical operation is compromised, market management can be override by the DSO through the technical operation control hierarchy described in Fig. 1. For these abnormal or emergency conditions, it makes sense to adapt the MG [17] and MMG [18] concepts. In fact, the $\mathrm{MG}$ and the $\mathrm{MMG}$ already contemplate the existence of a hierarchical monitoring and management solution that includes a suitable communications infrastructure, capable of managing the presence of EVs, either individually connected at the LV level or as a cluster of EVs (fleet charging station or fast charging station cases) connected at the MV level. Within an LV MG, an MGCC may control EV batteries through the VC.

As depicted in the "Technical Operation" column of Fig. 1, within an MMG environment, the elements of the MV grid, including MG and CVC, can be technically managed by a control entity, named CAMC, to be installed in the HV/MV substation. All the CAMC will be under the supervision of a single DMS, which is directly controlled by the DSO. It is important to stress that, in abnormal system operation conditions or in emergency modes, all the technical management and control tasks are a responsibility of the DSO, being performed by a main control entity, the DMS, and by the other distributed entities: CAMC and MGCC [18].

\section{TECHNICAL IMPACTS OF ELECTRIC VEHICLES INTEGRATION ON A MEDIUM VOLTAGE NETWORK}

As mentioned previously, a massive deployment of EV connections into the electricity distribution grid may bring 
some grid operation problems, such as branch congestions or large voltage drops.

Distribution grids are planned to be able to accommodate all the loads in the system, given a certain simultaneity factor, for the peak hour. With the integration of EVs into the network, congestion problems may take place if EVs follow a free charging policy instead of being managed and controlled under a conceptual framework similar to the one developed in this work. If this type of approach is not adopted, large investments in grid reinforcement may be needed.

Preliminary studies regarding this issue have been performed in some distribution grids. In this section, the results of the impacts provoked by the EVs on a typical MV distribution grid are presented, as well as an enhanced charging strategy developed to allow the integration of a larger number of EVs in the same grid, without proceeding to any infrastructure reinforcements.

\section{A. Medium Voltage Grid Architecture}

Fig. 2 describes the MV network used in this research. It corresponds to a typical semi-urban, $15-\mathrm{kV}$ grid, which despite being meshed is explored using a radial configuration (the dashed branches are open). It has two feeding points, represented by the round shapes in the figure, energizing two separated areas. The areas are separated by normally open branches marked in the figure. The specified voltage in the feeding points is 1.05 p.u.

In order to perform a 24-h simulation, a typical daily load diagram for a semi-urban MV grid was used. This

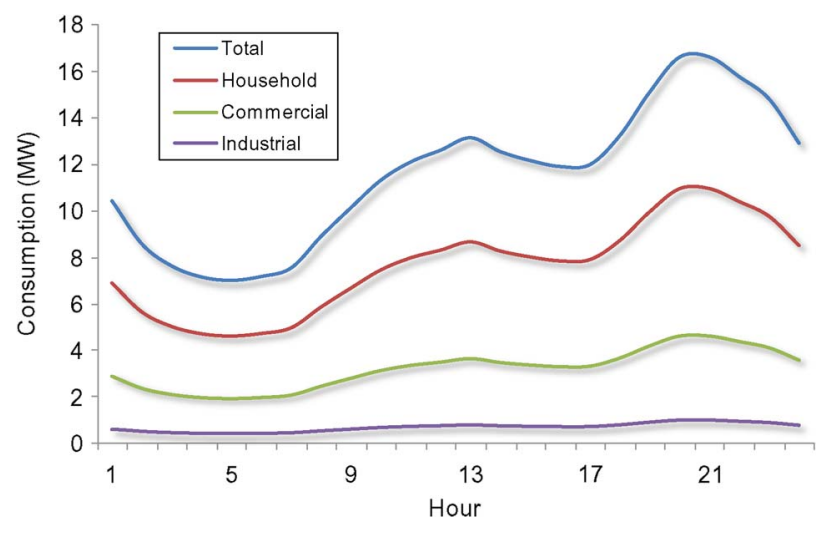

Fig. 3. Load profile during a typical day.

diagram, depicted in Fig. 3, was obtained by aggregating load diagrams of different types of Portuguese consumers.

\section{B. Electric Vehicles Characterization and Modeling}

The total number of vehicles considered to be related to the geographical area of this grid is approximately 12700 , which was determined assuming an average value of 1.5 vehicles per household.

The EV fleet considered includes plug-in hybrid vehicles and two types of full electric vehicles, each one of them with a different rated power: $1.5 \mathrm{~kW}$ for the hybrid (with a battery capacity of $6 \mathrm{kWh}$ ) [19], $3 \mathrm{~kW}$ for the medium EV (12 kWh) [20], and $6 \mathrm{~kW}$ for the large EV

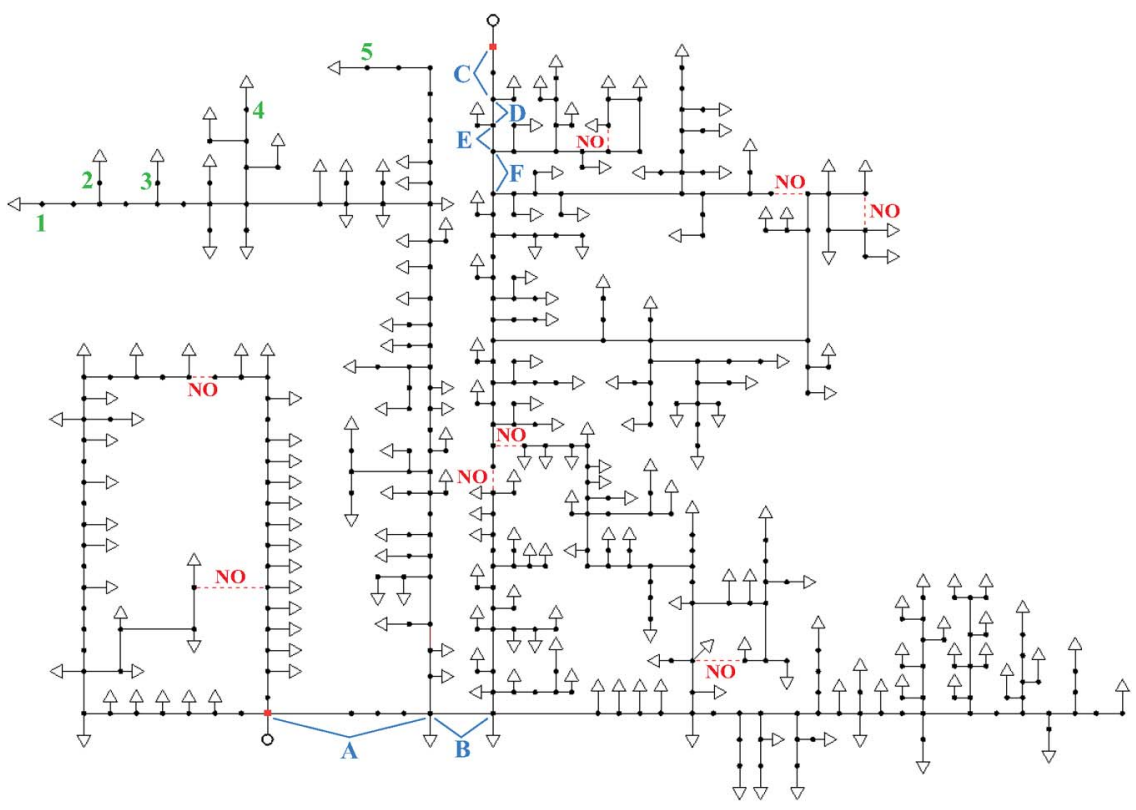

Fig. 2. Medium voltage distribution network (15 kV). The numbers 1-5 identify the buses that are more prone to having voltage problems. The letters A-F identify the most congested branches. (Normally Open branches are marked as NO.) 
(24 kWh) [21]. The three types of vehicles considered intend to represent cars with different driving ranges developed by automotive manufacturers to face different customers' needs. It was considered that the share of hybrids was $20 \%$ regarding the total number of EVs. The remaining $80 \%$ was equally split by the medium and large EVs.

The average charging time of each EV was assumed to be $4 \mathrm{~h}$ at their rated power, while their autonomy, regarding the energy consumption from the battery, was considered to be $30 \mathrm{~km}$ for the hybrid, $75 \mathrm{~km}$ for the medium $\mathrm{EV}$, and $150 \mathrm{~km}$ for the large EV (assuming a consumption of $0.20 \mathrm{kWh} / \mathrm{km}$ for the hybrid and $0.16 \mathrm{kWh} / \mathrm{km}$ for the medium and large EVs). Assuming that the average distance traveled by each vehicle is $35 \mathrm{~km}$ and that the hybrid runs roughly half of the distance in the electrical mode, the average charging frequency and charging energy should be $3.3 \mathrm{kWh}$ on a daily basis for the hybrid, $10.9 \mathrm{kWh}$ every two days for the medium EV, and $22.4 \mathrm{kWh}$ every four days for the large EV. However, due to the high number of uncertainties related with the drivers' behavior and the charging frequencies, it was considered a worst case scenario where all EVs charge their batteries on the same day.

\section{Dumb Charging Results}

The maximum number of EVs that could be safely integrated into the grid was determined using a dumb charging approach, meaning that EV owners are completely free to connect and charge their vehicles after parking, without any kind of charging control performed by the aggregator. The charging starts automatically when EVs plug-in and lasts for the next four hours. EVs were distributed through the grid proportionally to the residential power installed in each node.

The rationale to quantify the maximum share of EVs, when the dumb charging is adopted, was to increase their share in small steps until a grid violation occurs, for voltage values or power flow branches' congestion limits. The algorithm used for this procedure is described in Fig. 4.

Fig. 5 shows the EV load distribution attained with the algorithm described in Fig. 4, considering common Portuguese traffic patterns in residential areas [22] and assuming that all EV owners charge their vehicles when they arrive home from the last journey of the day, which provokes a large increase in load consumption that lasts for four hours. In this case, the allowable share of conventional vehicles that could be replaced by EVs was $10 \%$, since a grid restriction was attained for this level of EV penetration.

The impact assessment of EV integration on the voltage profiles and power lines' congestion levels of the selected MV distribution grid was made by using PSSE software to perform steady-state simulations. Power flow studies, for the load peak hour, were conducted for the base scenario (considering no EVs connected to the MV network) and for the scenario with $10 \%$ of EVs (assuming a dumb charging approach), in order to evaluate the changes in the voltages and in the branches' congestion levels. To assess the daily

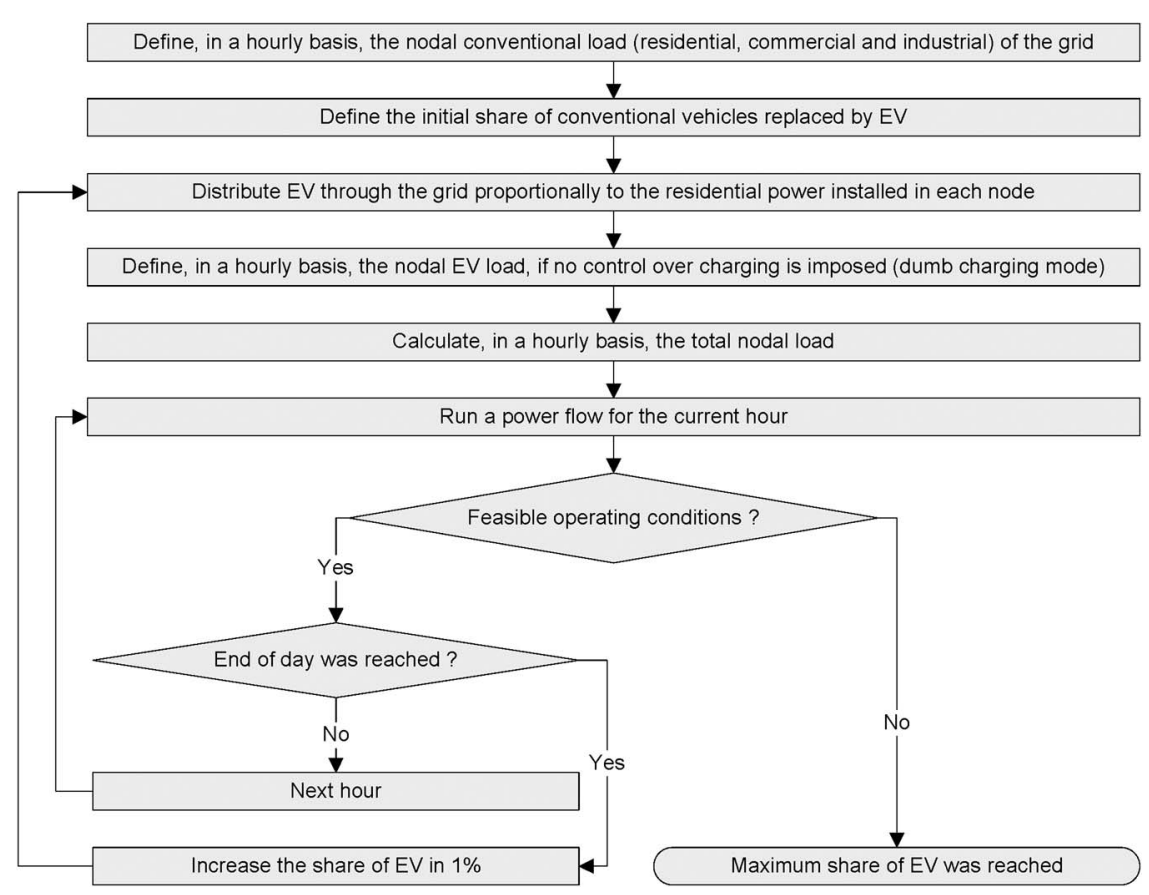

Fig. 4. Algorithm developed to quantify the maximum number of EVs that can be safely integrated into the grid with the dumb charging (without grid reinforcements). 


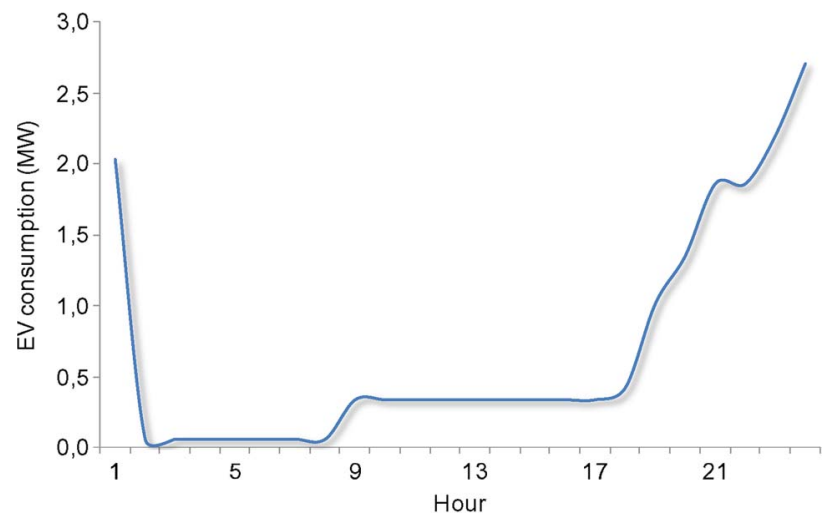

Fig. 5. EV consumption along a typical day for the dumb charging-10\% EV (MW).

losses, 24 power flows per scenario were performed. To establish a proper comparison between the two scenarios, the results regarding network impact assessment were compiled in Tables 1 and 2 and Figs. 5 and 6.

Looking at Table 1 it is possible to evaluate the effects of EV penetration in the voltage profiles of some buses electrically distant from the feeding points, for the peak hour. The remaining buses missing in Table 1 suffer similar changes in their voltages, but the five presented are those with bigger voltage drops.

As one can observe, voltage lower limit (0.95 p.u.) is almost reached in the scenario with $10 \%$ of EV. Thus, this percentage represents the feasible limit of EV integration with the dumb charging approach.

Table 2 shows the congestion levels evolution in six of the most loaded line sections within the grid, for the most congested hour.

The value of the daily losses is $10.21 \mathrm{MWh}$ for the scenario without EV and $11.78 \mathrm{MWh}$ with $10 \%$ of EV.

Fig. 6 shows the impact of $10 \%$ of EVs in the grid load diagram.

\section{Implementation of Enhanced Charging Strategies to Integrate a Larger Number of Electric Vehicles}

As shown in Figs. 3 and 5, the load peak hour in a residential area coincides with home arrival of a large number of EVs, following the common traffic patterns. Therefore, existing EVs will be plugged-in by that time. Having no control actions whatsoever, charging will start

Table 1 Voltage Levels_-Dumb Charging

\begin{tabular}{|lcccccc|}
\hline \hline \multirow{2}{*}{$\begin{array}{l}\text { Peak } \\
\text { Hour }\end{array}$} & Bus & 1 & 2 & 3 & 4 & 5 \\
\cline { 3 - 7 } & No EV & 0.961 & 0.962 & 0.962 & 0.962 & 0.964 \\
\hline 21 & No of EV & 0.951 & 0.951 & 0.951 & 0.951 & 0.953 \\
21 & $10 \%$ \\
\hline \hline
\end{tabular}

Table 2 Branches' Congestion Levels-Dumb Charging

\begin{tabular}{|c|c|c|c|c|c|c|c|}
\hline \multirow{2}{*}{$\begin{array}{l}\text { Peak } \\
\text { hour }\end{array}$} & \multirow[b]{2}{*}{ Line section } & \multicolumn{6}{|c|}{ Line ratings (\%) } \\
\hline & & A & B & $\mathrm{C}$ & D & E & $\mathrm{F}$ \\
\hline 21 & No EV & 71.7 & 63.5 & 43.2 & 43.1 & 42.9 & 35.1 \\
\hline 21 & $10 \%$ of $E V$ & 80.1 & 71.4 & 49.6 & 49.3 & 49.2 & 39.4 \\
\hline
\end{tabular}

immediately aggravating the previously existent operating conditions, as the results presented in the previous section demonstrate. It was possible to observe that the flow patterns on the branches near the feeding points suffer a considerable increase. Voltages, by their turn, suffer significant drops reaching, in some cases, the lower limit.

By analyzing these results it is easy to understand that the distribution network can handle, up to $10 \%$, EV penetration without changes in the usual electricity grid operation and planning procedures. This shows that grid restrictions may limit the growth of EV penetration, if no additional measures are adopted. Additionally, the increase in load consumption at peak hours, due to the presence of EVs, will require generation levels to increase [12], leading to a subsequent increase on electricity prices [8].

Nevertheless, different approaches can be adopted to deal with this problem, allowing the integration of a higher share of EVs while avoiding capital expenditures by the utility in network reinforcements.

Two solutions have been tested for this purpose: a simple dual tariff policy system and a more complex approach, based on a smart charging mechanism, to be performed by the DSO, in order to manage grid problems like high congestion levels or large voltage drops.

The dual tariff policy intends to simulate a situation where electricity is cheaper during some specific hours of the day. The aggregator might implement this approach by making specific dual tariff contracts with EV owners willing to take advantage of cheaper electricity price periods, similarly to what already happens between electricity traders and their clients. For this research, it was

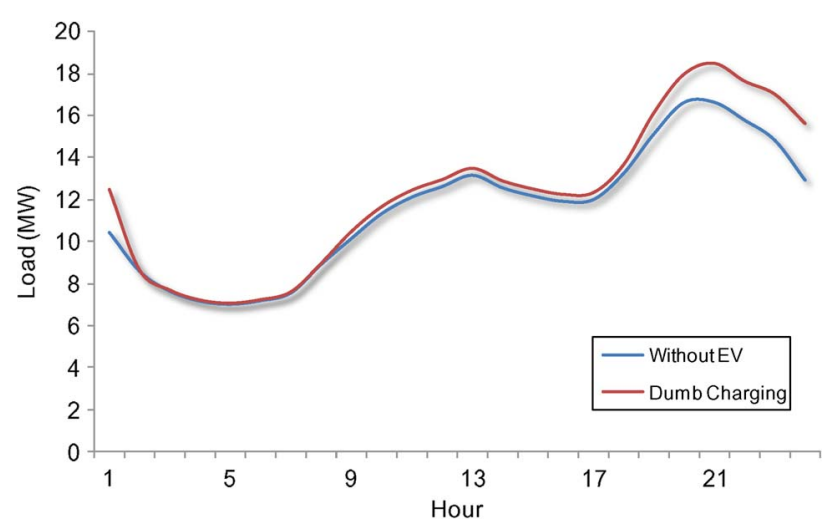

Fig. 6. MV grid load diagram with $10 \%$ EV. 
considered that the cheaper electricity periods are comprehended between $24-8 \mathrm{~h}$ and between $23-24 \mathrm{~h}$. It was also assumed that the economic incentive provided by this policy was enough to make $25 \%$ of the EV owners to adhere to the dual tariff policy. Thus, $75 \%$ of the EV owners will charge their vehicles according to the dumb charging load profile and 25\%, randomly chosen, will shift their consumption towards the cheaper period, meaning that $25 \%$ of the EVs will start charging at $23 \mathrm{~h}$.

The smart charging algorithm is presented in the shaded area of Fig. 7. The DSO might use it as an operational tool to define with the aggregator, prior to the market negotiations, the amount of EV load that needs to be shifted, in order to guarantee a safe operation of the

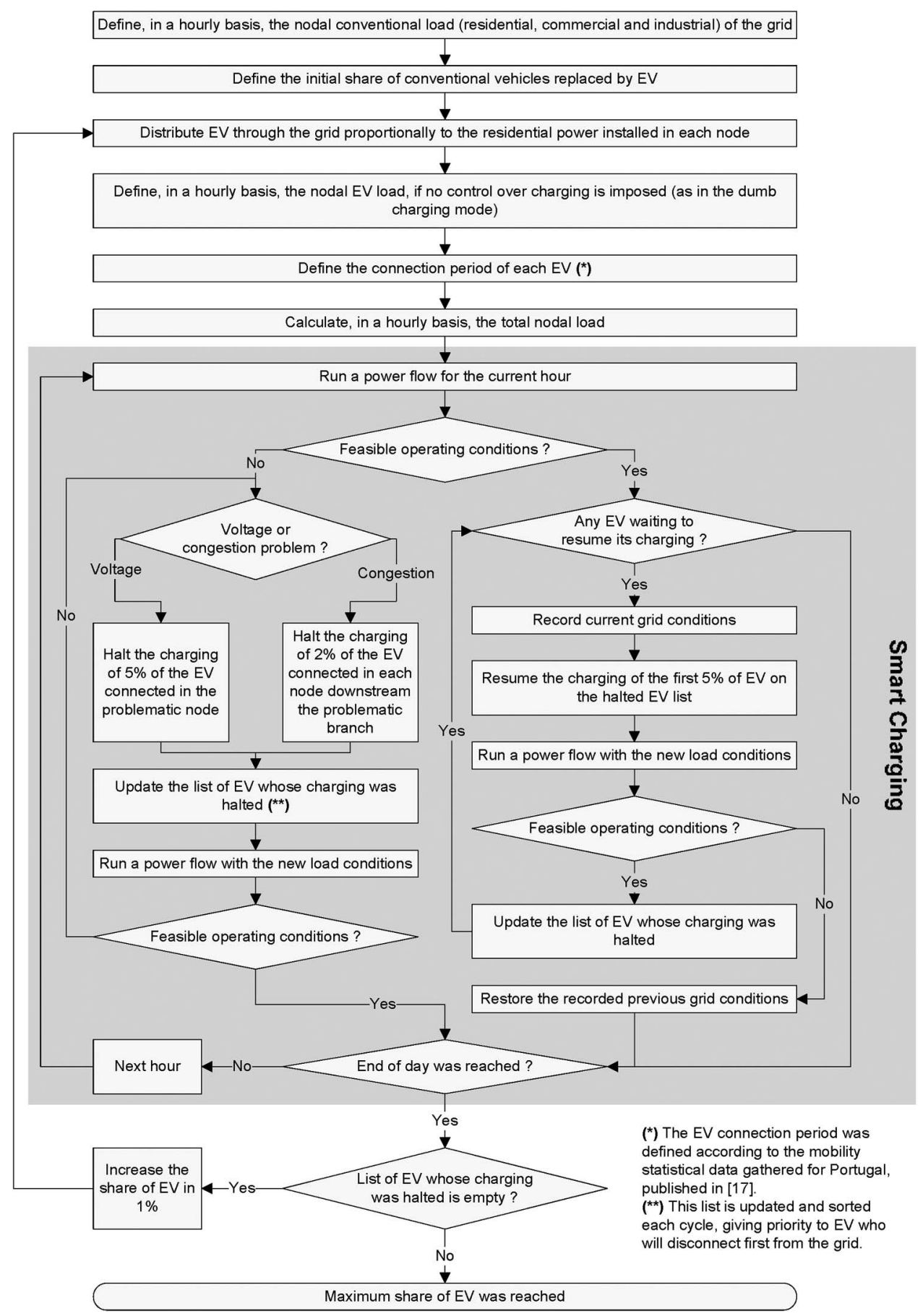

Fig. 7. Algorithm developed to maximize the number of EVs that can be safely integrated in the grid with the smart charging (without grid reinforcements). 


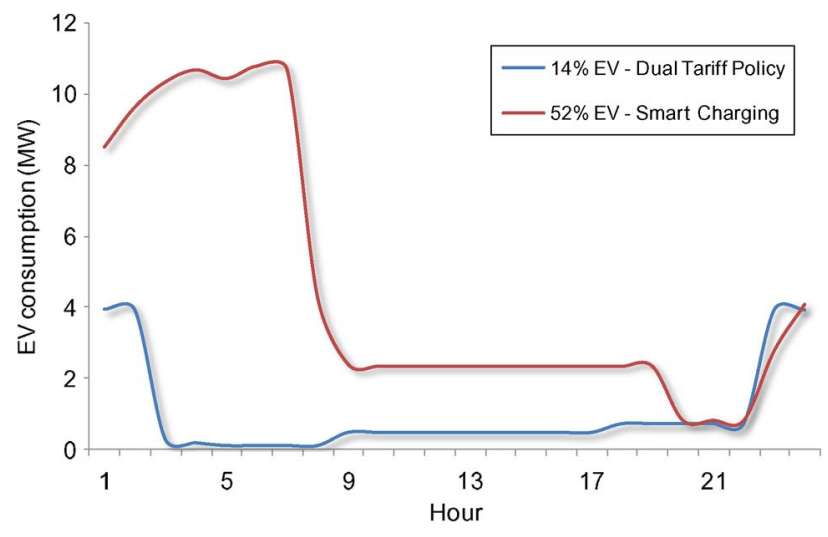

Fig. 8. EV power consumption along the day for the dual tariff policy and for the smart charging.

distribution network in the next day, especially during the peak hours.

The full algorithm presented in Fig. 8, which includes the smart charging (shaded area), was used to assess the maximum EV integration capacity of the grid, when this charging approach is implemented. The external loop, added to the smart charging algorithm, can lead to a network planning tool.

As is obvious, the smart charging approach will only be effective while the grid has enough capacity to provide all the power required by EVs. If the number of EVs keeps growing, there will be a moment in time where the reinforcement of the network infrastructures will be inevitable, even when the smart charging is applied.

\section{E. Dual Tariff Policy and Smart Charging Results}

The EV load profiles obtained, with the dual tariff policy and the smart charging algorithms, during one entire day, are presented in Fig. 8.

The allowable share of EVs that could be integrated into the MV network was 14\% with the dual tariff policy and $52 \%$ with the smart charging. The results for the smart charging were obtained by shifting, roughly, the charging period of $50 \%$ of the EVs. This means that the integration of $52 \%$ of EVs would require the aggregator to act over $50 \%$ of EVs, shifting their charging from the peak to the valley hours, in order to keep the grid operating within its technical limits.

Table 3 shows the effects of EV penetration in the voltage profiles of the same buses that were analyzed in the dumb charging scenario.

The results of the scenario with $10 \%$ of EVs, with the dumb charging, are presented again in Tables 3 and 4 only for comparison purposes.

The average voltage drop in the five buses is $1.1 \%$ for the dual tariff policy scenario and $1.2 \%$ for the smart charging scenario. This is a remarkable result given that in
Table 3 Voltage Levels

\begin{tabular}{|ccccccc|}
\hline \hline \multirow{2}{*}{ Peak } & & \multicolumn{5}{c|}{ Voltage (p.u.) } \\
\cline { 3 - 7 } Hour & Bus & 1 & 2 & 3 & 4 & 5 \\
\hline 21 & Without EV & 0.961 & 0.962 & 0.962 & 0.962 & 0.964 \\
21 & $10 \%$ EV - Dumb Charging & 0.951 & 0.951 & 0.951 & 0.951 & 0.953 \\
23 & $14 \%$ EV - Dual Tariff & 0.951 & 0.952 & 0.952 & 0.952 & 0.953 \\
23 & $52 \%$ EV - Smart Charging & 0.950 & 0.950 & 0.950 & 0.950 & 0.952 \\
\hline \hline
\end{tabular}

the smart charging scenario the integration level of EVs is more than the triple of the dual tariff policy one.

Bus 1 voltage for the dual tariff policy scenario, with $14 \%$ of EVs, almost reaches the lower limit, which is 0.95 p.u.. Thus, $14 \%$ EV integration represents the limit for the dual tariff approach. Voltage lower limit is also reached for the smart charging scenario, which shows that $52 \% \mathrm{EV}$ integration represents the limit for the smart charging approach. This shows that voltage, in this specific case, is the limiting factor to higher levels of EV integration. For the same integration level, voltage lower limit is reached first than branches maximum rating.

As one can observe, the smart charging allows attaining the highest EV penetration level without violating voltage lower limit. For lower integration levels, the dual tariff policy has a positive impact on the grid performance, but, for higher ones, it is not a good strategy since it concentrates a high number of EVs charging simultaneously around $23 \mathrm{~h}$, which provokes a large decrease in voltage values.

Table 4 shows the congestion levels evolution, in the same six line sections presented in Table 2 .

The average increase in the congestion levels of the six line sections is $13.0 \%$ for the dual tariff policy scenario and $20.8 \%$ for the smart charging scenario.

Even though this is not the most critical aspect of this network, branches' congestion is also an issue that deserves special attention given that it can be the limiting factor to higher levels of EV integration in networks with different characteristics from the one analyzed in this study.

Fig. 9 shows an overall comparison of branch loading in the peak hour of the scenarios without EVs (upper-left picture) and with 52\% of EVs (the remaining three), in order to provide a qualitative and general overview of the three charging methods' impact in this matter.

Fig. 10 shows the absolute values of the losses for the selected day (bars), referred to the left vertical axis, and

Table 4 Branches' Congestion Levels

\begin{tabular}{|cccccccc}
\hline \hline \multirow{2}{*}{$\begin{array}{c}\text { Peak } \\
\text { Hour }\end{array}$} & Line section & A & B & C & D & E & F \\
\cline { 3 - 7 } & Without EV & 71.7 & 63.5 & 43.2 & 43.1 & 42.9 & 35.1 \\
21 & $10 \%$ of EV - Dumb Charging & 80.1 & 71.4 & 49.6 & 49.3 & 49.2 & 39.4 \\
23 & $14 \%$ of EV - Dual Tariff & 80.0 & 71.7 & 50.6 & 50.3 & 50.1 & 39.5 \\
23 & $52 \%$ of EV - Smart Charging & 81.7 & 74.4 & 54.6 & 54.2 & 54.1 & 40.6 \\
\hline \hline
\end{tabular}


(a)

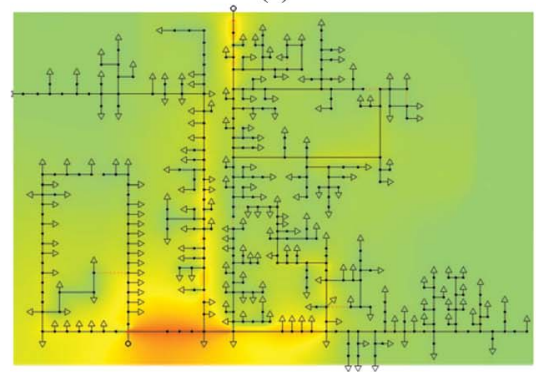

(c)

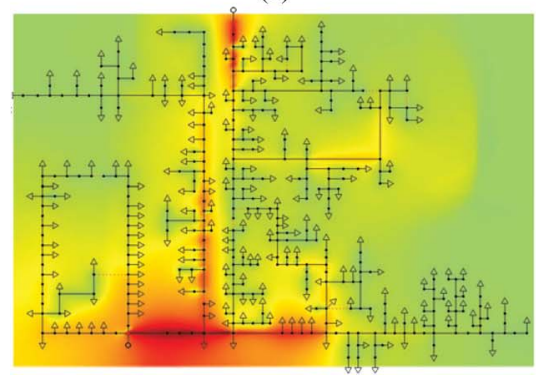

(b)

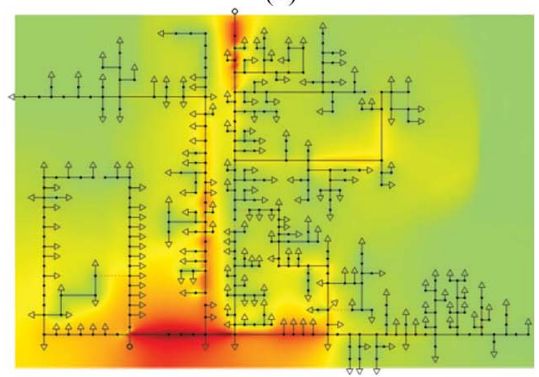

(d)

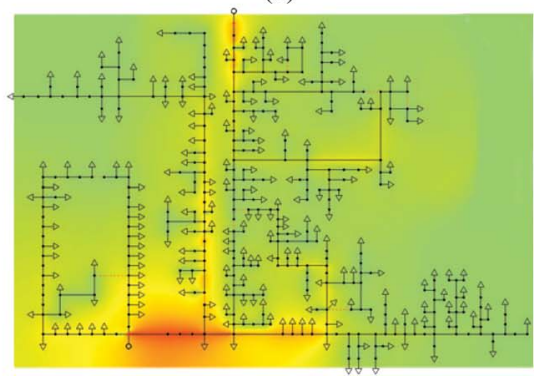

Fig. 9. Branches congestion levels at the peak hour (a) for the scenario without EV, and (b) for the scenario with $52 \%$ of EV with the dumb charging, (c) dual tariff policy, and (d) smart charging. Grading between green and red stands for increasing values of congestion, from $0 \%$ to $100 \%$.

their value relative to the overall energy consumption (crosses), referred to the right vertical axis.

As expected, the smart charging method is the one that provides better results since it makes the load distribution along the day more uniform, consequently reducing the grid's peak demand. The peak periods are the most critical for the losses as they are proportional to the square of the current, which is very high in such conditions.

In order to guarantee acceptable robustness levels and proper dimensioning of HV/MV distribution substations, network planning needs to take into account the peak load, which is the moment where the system is subjected to the

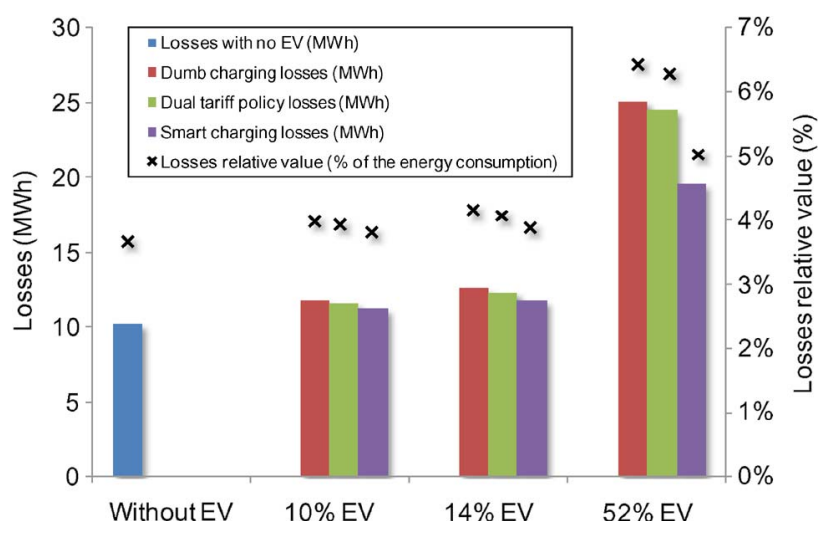

Fig. 10. Losses absolute values for the selected day (bars), referred to the left vertical axis, and their value relative to the overall energy consumption (crosses), referred to the right vertical axis. more demanding operating conditions. Thus, it is of utmost importance to analyze the impact of EV charging into the grid at the peak hour.

Table 5 presents the peak load, in MW, for all the scenarios considered and for all the charging methods implemented.

With the dumb charging approach, the load in the peak hour increases $85 \%$, from the scenario without EVs to the scenario with $52 \%$ of EVs, whereas with the dual tariff policy it increases $96 \%$. When the smart charging method is applied the peak load only increases $11 \%$, which is an outstanding achievement considering that $52 \%$ of EVs represent around 6500 vehicles demanding $111 \mathrm{MWh}$ (40\% of the network daily energy consumption) during one day. The bold values presented in the table are the peak load values obtained, for each charging method, when the maximum allowable percentage of EVs is reached.

The network load diagrams obtained for the scenario with $52 \%$ of EVs are presented in Fig. 11.

It is interesting to notice that the peak hour for the dumb charging approach changes from 21 to $24 \mathrm{~h}$, whereas with the dual tariff policy and the smart charging it changes from 21 to $23 \mathrm{~h}$.

\section{IMPROVING SYSTEM DYNAMIC OPERATION}

There are also several potential benefits from the integration of EVs in the electric power system dynamic behavior. From the grid perspective, an EV battery may be regarded as an extra storage device when plugged-in, but, more 
Table 5 Peak Load

\begin{tabular}{|lcccc}
\hline \hline & \multicolumn{4}{c}{ Peak load (MW) } \\
\cline { 2 - 5 } & No EV & Dumb charging & Dual tariff policy & Smart charging \\
\hline Without EV & $16.60(21 \mathrm{~h})$ & & & \\
$10 \%$ of EV & & $\mathbf{1 8 . 4 6}(\mathbf{2 1 h})$ & $17.50(23 \mathrm{~h})$ & $16.73(21 \mathrm{~h})$ \\
$14 \%$ of EV & $19.31(21 \mathrm{~h})$ & $\mathbf{1 8 . 7 3}(\mathbf{2 3 h})$ & $16.79(21 \mathrm{~h})$ \\
$52 \%$ of EV & $30.66(24 \mathrm{~h})$ & $32.55(23 \mathrm{~h})$ & $\mathbf{1 8 . 4 3 ( 2 3 h )}$ \\
\hline \hline
\end{tabular}

importantly, as a flexible and highly controllable load. Within the envisioned grid management and market operation framework, reserves can also be delivered by EVs.

However, depending on the type of reserve being provided EV will rely differently on the whole control infrastructure and its different market players. In this paper, the definitions for primary, secondary, and tertiary reserves are those present in the UCTE Operation Handbook [23].

For primary frequency control, EV owners will communicate their willingness to participate in primary reserve delivery to the aggregator, via the MGAU. Once this communication is established, the EV will be flagged as primary reserve provider and the control will be locally activated. When leaving the service provision, the EV again sends a signal to the aggregator and, if within a new regulatory framework where this service can be paid, the EV will get remunerated according to the period of time when the service was provided.

For secondary control, the AGC operation will be the centerpiece in the control hierarchy. The TSO, who is responsible for the AGC, will acquire in the electricity markets the secondary reserves that it needs from GENCO and aggregators. Then, in accordance with the secondary reserve services negotiated in the market with the TSO, the aggregator will send set points to the EV willing to

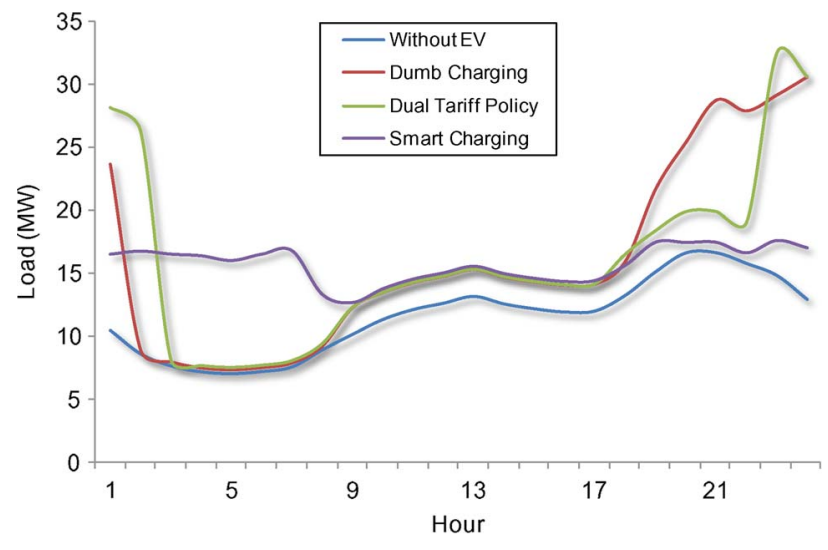

Fig. 11. Network load diagram for the scenario with $52 \%$ of EVs with the three charging strategies studied. provide this service. The set points that EVs will receive from the aggregator will lead to a load charging adaptation for the period of time the AGC requires this service.

Regarding the dynamic operation of an isolated system, due to its reduced robustness, the referred EV flexibility may be used to perform primary frequency control leading to an improvement of the global system's dynamic behavior. It would enable further deployment of intermittent RES in islands where the resilience of the system is the limiting factor to its integration. Conversely, in an interconnected system, the robustness of operation is high and frequency deviations are too small to be detected at the EV level. So, primary control is not of upmost importance, being secondary and tertiary reserves provision the main focus. By providing secondary reserve, EVs are avoiding expensive and more polluting generation groups that may compose the tertiary reserve to be started, in response to the full usage of traditional secondary reserve.

Moreover, as described before in this paper, EV integration concepts may be combined with other concepts like those of the MG and MMG working in a cooperating mode towards the new energy paradigm. Within this context, EVs decrease the need for dedicated storage, facilitating the deployment of the concept by decreasing cost of investment and improving the system's performance when operating in emergency modes.

This section presents two studies related with primary frequency control for both emergency operation of an MG and for a physical island, while providing an introductory topic on the modeling of EV grid interfaces (VC) for these cases.

\section{A. EVs Grid Interface Modeling}

To enable all the envisioned modes of operation, a proper electronic interface control should be adopted, different from a simple diode bridge usually adopted for these purposes. This interface must be able to receive set points to perform centralized control actions, such as secondary reserve provision, and adapt EV operating conditions as a reaction to local indicators, e.g., for primary reserve provision. Evidently, the damping provided by EV load will be highly dependent upon the EV parked population, varying greatly throughout the day, and will require parameter tuning, for instance, in AGC. 


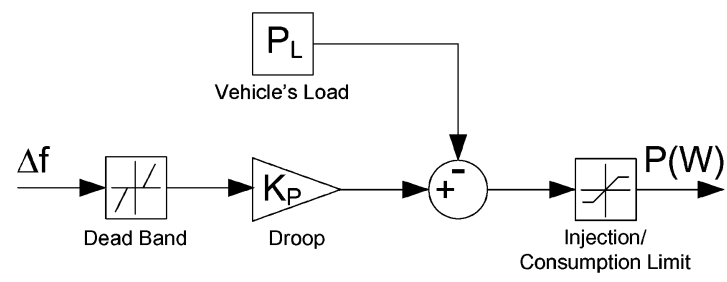

Fig. 12. Control loop for EV active power set point.

Being the system frequency an instantaneous indication of the power balance in the network, it must therefore be used to adapt the active power charging/discharging of the EV batteries. The implementation of this approach requires a phase-locked loop (or equivalent method) to measure frequency deviations at each EV grid interface. However, the delays introduced by such algorithms are quite reduced and do not affect the efficacy of the control approach [24]. A frequency control droop loop [25] can then be used to adjust the active power set point of an EV inverter interface (Fig. 12), which is a part of the VC. In this way, a smart EV grid interface, capable of responding locally to frequency changes, is adopted, instead of a dumb battery charging solution.

To this conventional control method, a dead band, where EVs do not respond to frequency deviations, should be added to guarantee longevity of the batteries and thus a beneficial synergy between parties: the grid operator and the EV owners. This dead band, as well as the slopes of the droops, should be defined according to the composition of the system, as well as the EV owners' willingness to help with system frequency regulation. After several tests for these case studies, a $0.1-\mathrm{Hz}$ dead zone was used and an $\mathrm{MW} / \mathrm{Hz}$ droop was defined.

As EV batteries, under a V2G concept, can either absorb or inject active power, a saturation block with upper and lower limits must be added. A block providing the steady-state set point of active power must also be included, working as an offset to the droop. This block represents EV normal consumption status.

Fig. 13 shows schematically the droop configuration that can be implemented for this EV grid interface control strategy. For frequency deviations larger than the defined dead band, the EV battery will respond according to one of the given slopes. If frequency suffers a negative deviation, then the battery charging will, first, reduce its power consumption and, if frequency decreases further, it will inject power into the grid. On the contrary, if there is a positive deviation, then the battery will increase the power absorbed from the grid. The zero-crossing frequency $f_{0}$ and the frequency at which maximum power is injected to the grid $f_{\text {neg }}$, are parameters of free choice that can be set according to the required slopes and operation modes (controllable load or V2G). $f_{\text {neg }}$ only exists when EVs are working in V2G mode of operation.

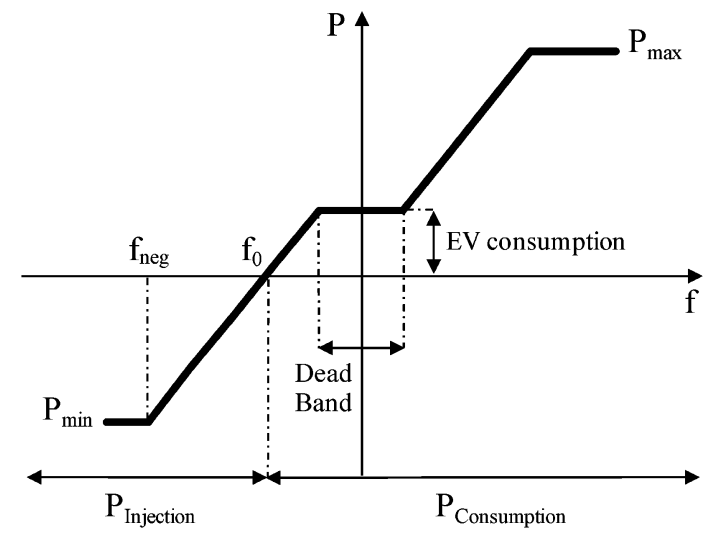

Fig. 13. Droop control for vc.

\section{B. Microgrid Operation}

Since EVs provide additional storage capability and a large degree of controllability during charging it can be assumed that an LV MG can be operated in islanding mode with enhanced performance.

Therefore, the control strategy defined in the previous section was tested in the MG presented in Fig. 14, using

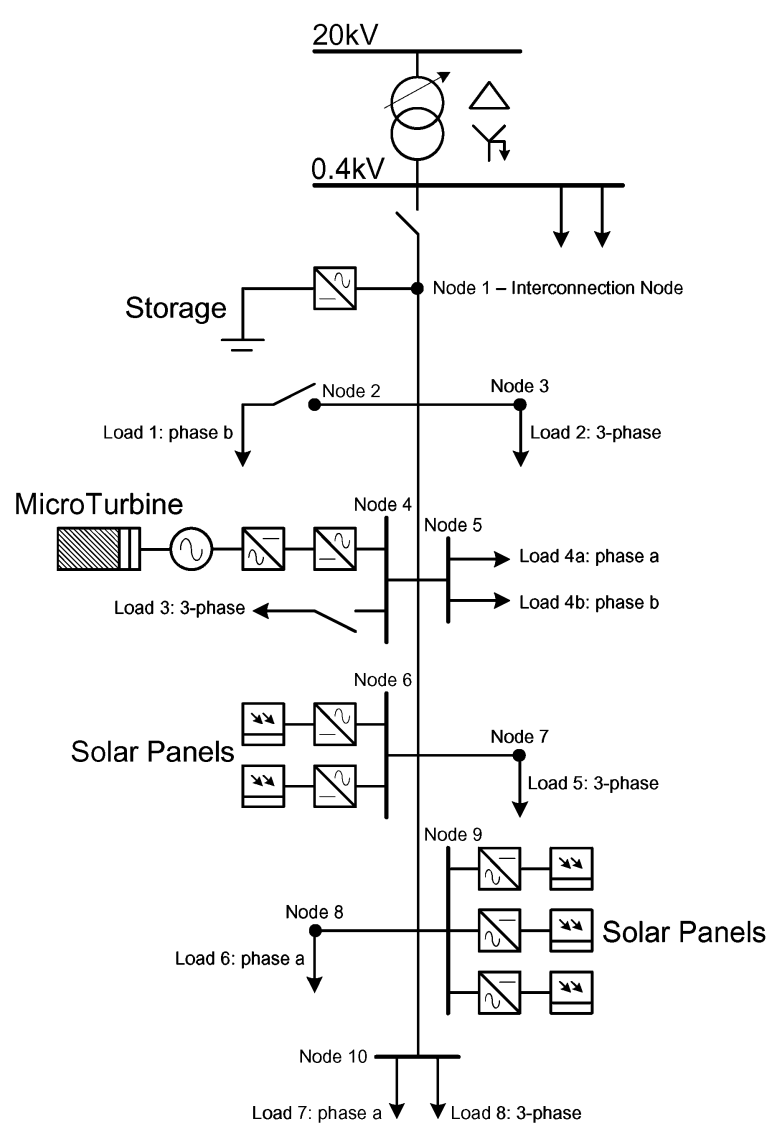

Fig. 14. Single line diagram of the investigated MG. 
the Matlab/Simulink simulation platform described in [17]. This MG is composed of a central storage device that is the main frequency control unit for islanded operation, several microsources (microturbines and PV solar panels) as well as controllable loads. To evaluate the MG primary frequency control performance using this control approach, one single-phase (L1) and one three-phase (L3) loads were disconnected (L1 at $t=5 \mathrm{~s}$ and L3 at $t=2 \mathrm{~s}$ ) and reconnected (L1 at $t=10 \mathrm{~s}$ and L3 at $t=8 \mathrm{~s}$ ). The effect on the grid frequency is shown in Fig. 15. The initial frequency drop is due to islanding (local load is larger than generation): it was assumed that for $t \leq 0 \mathrm{~s}$ the MG is in the interconnected mode, and so the frequency is $50 \mathrm{~Hz}$.

In Fig. 15(a), since over frequencies are dominant, one can conclude that during most of this time interval, generation exceeds the load. It would be therefore possible to charge the storage devices, including EVs, at least in the low load period. If EVs are connected to the grid with a fixed charging rate, the frequency shifts to a lower level, as can be seen in Fig. 15(b). In Fig. 15(b), the MG main storage device unit has to inject energy in the grid during most of the time, to compensate the load/generation mismatch. Adapting dynamically the load of EV batteries when the frequency falls below $50 \mathrm{~Hz}$ would then be an efficient procedure, as it reduces the total required central storage energy capacity. In Fig. 15(c) and (d), it can be observed that system frequency can be influenced by choosing the value of the zero-crossing frequency $f_{0}$ and an excellent dynamic behavior was obtained in Fig. 15(d).

\section{Larger Isolated Systems}

Concerning a large isolated system, the research described in [11] evaluates the benefits of using V2G to maximize the integration of intermittent RES in an island.

This island is characterized by an electric load ranging from $1770 \mathrm{~kW}$ at the valley hours to $4200 \mathrm{~kW}$ at the peak hour. Its generation system is composed of four diesel units (two of $1500 \mathrm{~kW}$ and two of $1800 \mathrm{~kW}$, performing primary and secondary frequency regulation, as well as providing a voltage reference), two wind turbines (with $660 \mathrm{~kW}$ each), and mild PV penetration. The objective of this study was to evaluate the possibility of integrating an extra wind turbine without jeopardizing the dynamic behavior of the system. Considering an average of one vehicle per household, it was assumed that the local vehicle fleet is composed of 2150 vehicles, with 15\% EV integration level.

For the conventional diesel generators, a common fourth-order model was taken from the literature [26]. Frequency regulation is performed through conventional proportional and integral control loops. Wind generators were modeled as simple induction generators, as described in [26]. (a)

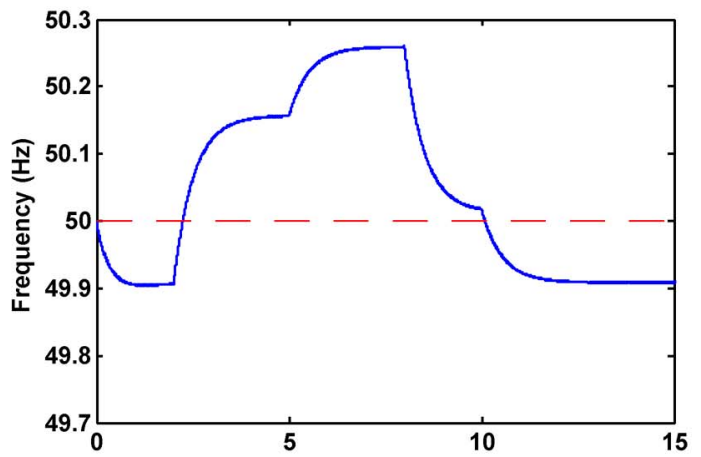

(c)

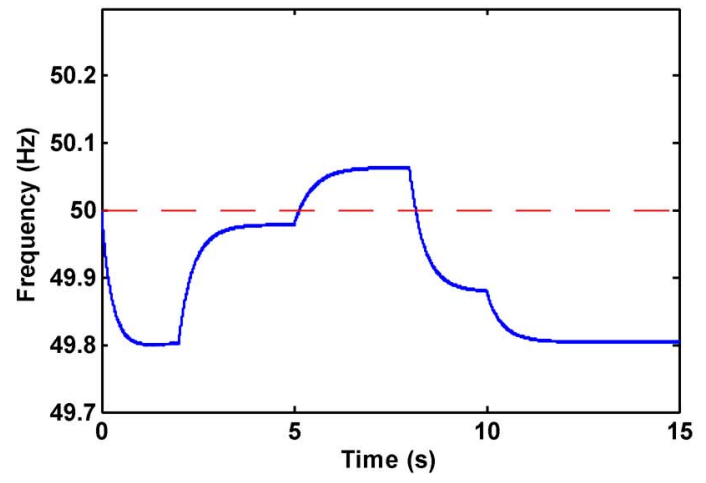

(b)

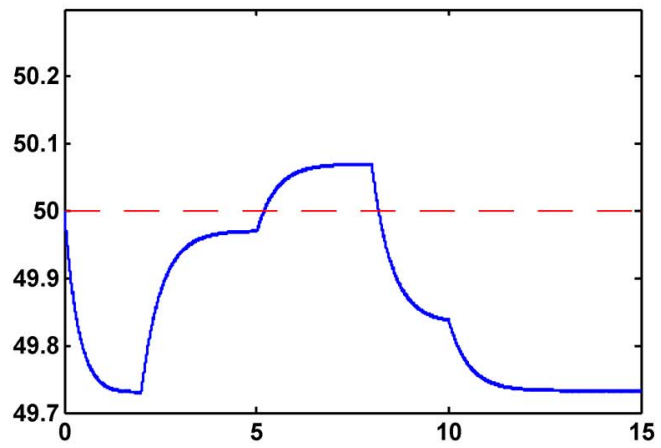

(d)

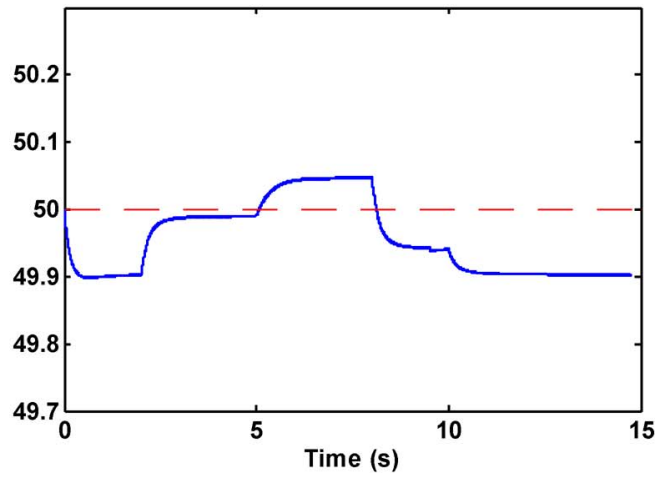

Fig. 15. Frequency evolution in the load following configuration. (a) Without EV. (b) EV with fixed charging rate. (c) EV with $f_{0}=49.5 \mathrm{~Hz}$. (d) EV with $f_{0}=49.9 \mathrm{~Hz}$. 
Using Matlab/Simulink, the system was assembled following the general principles used for dynamic stability studies in conventional power systems, which consist in keeping the model of the sources in the time domain (solution of a set of differential equations) and to use a steady-state frequency domain model to represent the electrical components and their links. The usual approach followed in conventional power systems dynamic studies was adopted, in which the fast transients are neglected [27], [28], being the electric network represented by its admittance matrix in the $\mathrm{d}-\mathrm{q}$ reference frame.

The methodology developed to evaluate this potential increase in wind generation, with EVs, is as follows.

1) The isolated system was characterized, in terms of available generation and load. All the components were modeled and connected in a single bus system.

2) EV penetration was then characterized and the model for EV grid connections, described in Section IV-A, was adopted (featuring primary frequency control).

3) A sudden decrease of wind power generation was simulated and its impact on the system's frequency was evaluated for two different situations: a) when EVs are in charge mode only and b) when they participate in primary frequency control. The period chosen to perform this study refers to a valley hour $(5 \mathrm{~h})$, where wind power represents a considerable share in the electricity being generated. Such period was selected as it represents a worst case scenario for frequency deviation issues. Due to the system's high dependency on the wind power production, if a sudden decrease in wind speed occurs, system frequency might drop to risky levels.

4) The amount of wind power generation (by increasing the wind power installed capacity) was

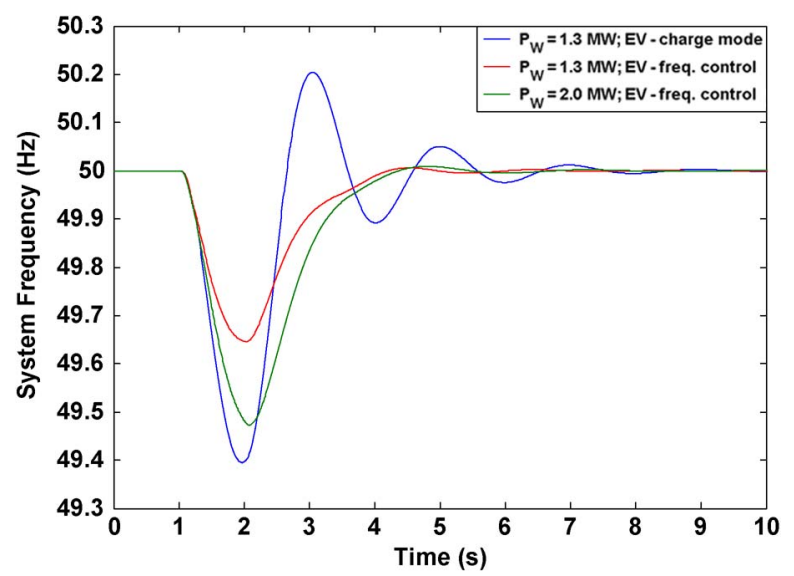

Fig. 16. System frequency response.

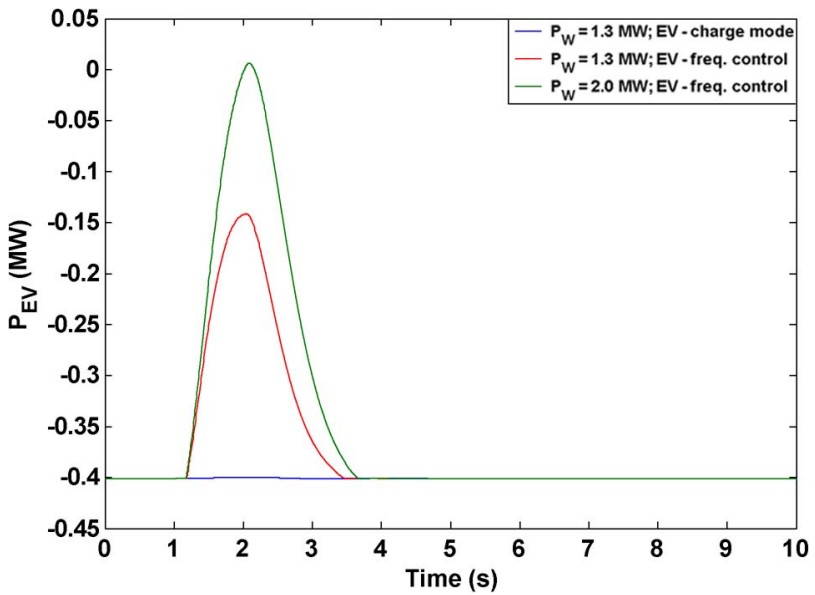

Fig. 17. EV active power consumption/injection.

increased and the feasibility of this new situation was assessed by repeating step 3).

The simulations were performed using a Matlab/ Simulink tool. The main results obtained, concerning frequency deviations following a sudden drop in wind speed from 9.5 to $6 \mathrm{~m} / \mathrm{s}$, are depicted in Fig. 16. In the original conditions the frequency dropped to $49.40 \mathrm{~Hz}$. In that case, only the diesel units are responsible for frequency control. Nevertheless, when EVs participate in frequency control, frequency drops only to $49.65 \mathrm{~Hz}$ and also the preexisting overshoot will cease to occur.

When EVs participate in frequency control, then the low damped situation that is verified at the charging only mode disappears, as EV inverters have very small response delays. So, EVs interfaced with the grid through VC grant more stability to the system, just by reducing their level of consumption from 401 to $141 \mathrm{~kW}$, as shown in Fig. 17 during the frequency drop. This allows an initial slower reaction of the diesel units that increase their output as the EV load returns to normal and gradually replace the EV contribution. Another visible phenomenon is that when frequency starts to drop, in both cases, the system evolves equally. This is due to the dead band in the EV droop control that avoids premature wear of the batteries, but also prevents EV reaction to smaller frequency deviations.

Since frequency deviations can be largely damped when adopting this kind of smart EV grid interface, one can assume that wind generation can be increased without compromising power quality in terms of system frequency excursions. In order to evaluate the feasibility of this hypothesis an increase in wind power installed capacity was considered for the scenario under analysis.

To perform the verification of this premise, a third wind generator, similar to the others, was then assumed to be in operation in the system. In this case, the system frequency, when EVs are in charging mode only, drops to $49.13 \mathrm{~Hz}$ (not shown in the picture) and, with EVs participating in 
frequency control, to $49.47 \mathrm{~Hz}$. In the last case, EVs had to inject power into the grid (Fig. 17). Nevertheless, the global power injection required, $6 \mathrm{~kW}$, is very small.

So, it is possible to verify that this system improved dramatically its performance when EVs were used to help in frequency control. It was even possible to integrate one extra wind generation unit with improved system operation performance.

\section{CONCLUSION}

Large deployment of EVs will impose tremendous challenges to the operation and management of the electric power systems of the future. Such new scenario will require the development of an adequate technical and market operation framework, such as the one proposed in this work, in order to reduce the negative impacts that EVs might provoke and to increase their potential benefits. The real startup/demonstration of the electric mobility concept should involve, in a first stage, a progressive use of EVs by fleets of cars (taxis, transportation services, commercial services, municipal services) being gradually extended to the general public. This first stage will provide a way to learn and adjust solutions to massively extend the concept to the general public and to allow grid operators to improve the adopted solutions regarding the design, management, and control of the power system.

The results obtained from the case studies presented in this work show that the adoption of advanced centralized EV charging control strategies allows the integration of a larger number of EVs in the system, without the need to proceed to grid reinforcements. These charging schemes allow operating the networks in less stressed conditions, with improved voltage profiles and lower congestion levels. Additionally, the adoption of a local level of control, housed in the vehicle grid interface, will allow a better operation performance in islanded operation mode and a safe increase of the quantity of intermittent and variable RES (e.g., wind and photovoltaic) installed in isolated power systems, since EV batteries proved to be capable of providing fast compensation to the system.

Although not addressed in this work, transformer rating might also be a limiting factor for EV integration in distribution networks [9]. Transformers have the capability to be temporarily overloaded, provided they have a subsequent low loading period to decrease their temperature. For higher integration levels, the smart charging algorithm may be enhanced to take this into account.

\section{REFERENCES}

[1] W. Kempton and J. Tomic, "Vehicle-to-grid power fundamentals: Calculating capacity and net revenue," J. Power Sources, vol. 144, no. 1, pp. 268-279, Jun. 2005.

[2] M. Valentine-Urbschat and W. Bernhart, "Powertrain 2020 - The future drives electric," Roland Berger Strategic Consultants Rep., Munich, Germany, pp. 1-95. [Online] Available: http://www.rolandberger.com/

[3] M. Kinter-Meyer, K. Schneider, and R. Pratt, "Impacts assessment of plug-in hybrid electric vehicles on electric utilities and regional U.S. power grids. Part I-Technical analysis," Pacific North West National Lab., Richland, WA, PNNL-SA-61669, Jan. 2007.

[4] EPRI \& NRDC Environmental assessment of plug-in hybrid electric vehicles. Volume 1: Nationwide greenhouse gas emissions, Final Rep. 1015 325, Jul. 2007, pp. 1-56.

[5] P. Denholm and W. Short, "An evaluation of utility system impacts and benefits of optimally dispatched plug-in hybrid electric vehicles," Nat. Renewable Energy Lab. (NREL), Golden, CO, Tech. Rep., Oct. 2006.

[6] M. J. Scott, M. Kintner-Meyer, D. Elliott, and W. Warwick, "Economic assessment and impacts assessment of plug-in hybrid vehicle on electric utilities and regional U.S. power grids. Part II," Pacific North West National Lab., Richland, WA, PNNL-SA-61687, Jan. 2007.

[7] S. Hadley and A. Tsvetkova, "Potential impacts of plug-in hybrid electric vehicles on regional power generation," Oak Ridge Nat. Lab., Oak Ridge, TN, Rep. ORNL/ TM-2007/150, Jan. 2008.

[8] "Electrification roadmap: Revolutionizing transportation and achieving energy security,"
Electrification Coalition, Washington, DC, Nov. 2009. [Online]. Available: http://www. electrificationcoalition.org/

[9] C. Roe, F. Evangelos, J. Meisel, S. Meliopoulos, and T. Overbye, "Power system level impacts of PHEVs," in Proc. 42nd Hawaii Int. Conf. Syst. Sci., pp. 1-1, 2009.

[10] C. Guille and G. Gross, “A conceptual framework for the vehicle-to-grid (V2G) implementation," Energy Policy, vol. 37, no. 11, pp. 4379-4390, Nov. 2009

[11] J. A. P. Lopes, P. M. R. Almeida, and F. J. Soares, "Using vehicle-to-grid to maximize the integration of intermittent renewable energy resources in islanded electric grids," in Proc. Int. Conf. Clean Electr. Power Renewable Energy Resources Impact, Capri, Italy, pp. 290-295, Jun. 2009.

[12] J. A. P. Lopes, F. J. Soares, P. M. R. Almeida, and A. M. M. da Silva, "Smart charging strategies for electric vehicles: Enhancing grid performance and maximizing the use of variable renewable energy resources," in Proc. 24th Int. Battery Hybrid Fuel Cell Electr. Vehicle Symp. Exhib., Stavanger, Norway, pp. 1-11, May 2009.

[13] R. J. Bessa and M. A. Matos, "The aggregator agent for EV role in the electricity market," presented at the Med Power, Cyprus, Nov. 2010

[14] J. A. P. Lopes, F. J. Soares, and P. M. R. Almeida, "Identifying management procedures to deal with connection of electric vehicles in the grid," in Proc. PowerTech, Bucharest, Romania, pp. 1-8, Jun./Jul. 2009.

[15] A. Hajimiragha, C. A. Cañizares, M. W. Fowler, and A. Elkamel, "Optimal transition to plug-in hybrid electric vehicles in Ontario, Canada, considering the electricity-grid limitations," IEEE Trans. Ind. Electron., vol. 57, no. 2, pp. 690-701, Feb. 2010.

[16] K. Clement-Nyns, E. Haesen, and J. Driesen, "The impact of charging plug-in hybrid electric vehicles on a residential distribution grid," IEEE Trans. Power Syst., vol. 25, no. 1, pp. 371-380, Jan. 2010.

[17] J. A. P. Lopes, C. L. Moreira, and F. O. Resende, "Microgrids black start and islanded operation," in Proc. 15th Power Syst. Comput. Conf., Liege, Belgium, pp. 1-7, Aug. 22-26, 2005.

[18] N. J. Gil and J. A. P. Lopes, "Hierarchical frequency control scheme for islanded multi-microgrids operation," in Proc. IEEE Lausanne Power Tech., Lausanne, Switzerland, Jul. 2007, pp. 473-478.

[19] Serious Wheels, Venturi Specifications. [Online]. Available: http://www. seriouswheels.com/cars/2010/ top-2010-Venturi-Eclectic-Renderings.htm

[20] Green Car Congress, Audi A1 E-Tron Specifications. [Online]. Available: http:// www.greencarcongress.com/2010/03/ aletron-20100303.html

[21] Nissan, Nissan Leaf Specifications. [Online] Available: http://www.nissanusa.com/ev/ media/pdf/specs/FeaturesAndSpecs.pdf

[22] INE-Instituto Nacional de Estatística, "Inquérito à mobilidade da população residente," (in Portuguese), 2000. [Online]. Available: http://www.ine.pt/xportal/ xmain? $x$ pid=INE\&xpgid $=$ ine publicacoes\&PUBLICACOESpub_boui $=$ 7250963\&PUBLICACOESmodo $=2$

[23] European Network of Transmission System Operators for Electricity-ENTSO-E, UCTE Operation Handbook, Mar. 2009. 
[24] M. Mojiri, D. Yazdani, and A. Bakhshai, "Robust adaptive frequency estimation of three-phase power systems," IEEE Trans. Instrum. Meas., vol. 59, no. 7, pp. 1793-1802, Jul. 2010.

[25] A. Engler, "Applicability of droops in low voltage grids," Int. J. Distrib. Energy Resources, vol. 1, no. 1, pp. 3-15, Jan. 2005.
[26] P. Kundur, Power System Stability and Control. New York: McGraw-Hill, 1994.

[27] G. S. Stavrakakis and G. N. Kariniotakis, "A general simulation algorithm for the accurate assessment of isolated diesel-wind turbines systems interaction. Part I: A general multimachine power system model," IEEE Trans. Energy Conv., vol. 10, no. 3, pp. 577-583, Sep. 1995.
[28] G. S. Stavrakakis and G. N. Kariniotakis, "A general simulation algorithm for the accurate assessment of isolated diesel-wind turbines systems interaction. Part II: Implementation of the algorithm and case-studies with induction generators," IEEE Trans. Energy Conv., vol. 10, no. 3, pp. 584-590, Sep. 1995.

\section{ABOUT THE AUTHORS}

João A. Peças Lopes (Senior Member, IEEE) received the electrical engineering degree, the Ph.D. degree in electrical engineering, and the Aggregation degree from the University of Porto, Porto, Portugal, in 1981, 1988, and 1996, respectively.

Currently, he is Full Professor at the Department of Electrical Engineering, Faculty of Engineering, University of Porto, and Director of the Sustainable Energy Systems PhD course. He is also Director of the Instituto de Engenharia de Sistemas e Computadores do Porto (INESC), Porto, Portugal.

Filipe Joel Soares (Student Member, IEEE) received the Physics degree (five-year course) from the Faculty of Sciences and an Electrical Engineering (Renewable Energies) Postgrad from Porto University, Porto, Portugal, in 2004 and 2007, respectively, where he is currently working towards the Ph.D. degree in sustainable energy systems.
Currently, he is a Researcher in the Power Systems Unit, Instituto de Engenharia de Sistemas e Computadores do Porto (INESC), Porto, Portugal. His main research interests are focused on V2G impacts assessment.

Pedro M. Rocha Almeida (Student Member, IEEE) received the Electrical Engineering degree (five-year course) from the Faculty of Engineering, Porto University, Porto, Portugal, in 2007, where he is currently working towards the Ph.D. degree in sustainable energy systems.

Currently, he is a Researcher in the Power Systems Unit, Instituto de Engenharia de Sistemas e Computadores do Porto (INESC), Porto, Portugal. His main research interests are focused on V2G dynamics and control. 